\title{
Optical Spectroscopy of the Large Kuiper Belt Objects 136472 (2005 FY9) and 136108 (2003 EL61)
}

\author{
S. C. Tegler ${ }^{1}$ \\ Department of Physics and Astronomy, Northern Arizona University, Flagstaff, AZ, 86011 \\ Stephen.Tegler@nau.edu \\ W. M. Grundy \\ Lowell Observatory, 1400 W. Mars Hill Road, Flagstaff, AZ, 86001 \\ W. Romanishin ${ }^{1}$ \\ Department of Physics and Astronomy, University of Oklahoma, Norman, OK, 73019 \\ G. J. Consolmagno ${ }^{1,2}$ \\ Vatican Observatory, Specola, Vaticana, V-00120, Vatican City State \\ Department of Physics, Fordham University, Bronx, NY, 10458 \\ K. Mogren \\ Department of Physics and Astronomy, Northern Arizona University, Flagstaff, AZ, 86011 \\ and \\ F. Vilas ${ }^{1}$ \\ MMT Observatory, PO Box 210065, University of Arizona, Tucson, AZ, 85721
}

\begin{abstract}
We present high signal precision optical reflectance spectra of the large Kuiper belt objects 2005 FY9 and 2003 EL61. The spectrum of 2005 FY9 exhibits strong $\mathrm{CH}_{4}$-ice bands. A comparison between the spectrum and a Hapke model indicates
\end{abstract}

\footnotetext{
${ }^{1}$ Observer at the MMT Observatory. Observations reported here were obtained at the MMT Observatory, a joint facility of the University of Arizona and the Smithsonian Institution.

${ }^{2}$ 2006-2007 Loyola Chair, Fordham University
} 
the $\mathrm{CH}_{4}$ bands are shifted $3.25 \pm 2.25 \AA$ relative to pure $\mathrm{CH}_{4}$-ice, suggesting the presence of another ice component on the surface of $2005 \mathrm{FY} 9$, possibly $\mathrm{N}_{2}$-ice, CO-ice, or Ar. The spectrum of 2003 EL61 is remarkably featureless. There is a hint of an $\mathrm{O}_{2}$-ice band at $5773 \AA$; however, this feature needs to be confirmed by future spectroscopic observations of 2003 EL61 with a higher continuum signal precision, sufficient to detect a second weaker $\mathrm{O}_{2}$-ice band at $6275 \AA$.

Subject headings: Kuiper Belt — techniques: spectroscopic

\section{Introduction}

Measuring the surface composition of Kuiper belt objects (KBOs) may provide clues about the composition and environment of the primordial solar nebula as well as the important evolutionary processes occurring in the outer Solar System over the last 4.5 Gyr. Unfortunately, there are only a handful of KBOs that are known to exhibit ice absorption bands in their spectra. $\mathrm{H}_{2} \mathrm{O}$-ice bands are seen in spectra of 1996 TO66 (Brown et al. 1999), Varuna (Licandro et al. 2001), Quaoar (Jewitt \& Luu 2004), and Orcus (Fornasier et al. 2004a). $\mathrm{CH}_{4}$-ice bands are seen in spectra of Pluto (Cruikshank et al. 1976; Fink et al. 1980; Grundv \& Fink 1996), Neptune's satellite Triton, which may be a captured KBO (Cruikshank et al. 1993), Eris (Brown et al. 2005), and 2005 FY9 (Licandro et al. 2006)

- There are perhaps a dozen objects that exhibit spectra with no ice absorption bands (Doressoundiram et al. 2003; Fornasier et al. 2004b)

The recent discovery of extraordinarily bright and large KBOs opens up a new opportunity for the physical and chemical studies of KBO surfaces with high signal precision optical reflectance spectroscopy. Bright objects make it possible to either carry out in-depth physical modeling of ice and mineralogical absorption bands or set stringent upper limits on the presence of such bands.

Here we present high signal precision optical reflectance spectra of KBOs 2005 FY9 and 2003 EL61. Both objects are candidates for membership in the newly defined class "dwarf planet." The diameter of 2005 FY9 is estimated at $1600 \mathrm{~km}$ (Brown et al. 2006) while 2003 EL61 appears to be a highly elongated ellipsoid with axes of $1950 \times 2500 \mathrm{~km}$ (Rabinowitz et al. 2006). For comparison, Pluto has a diameter of $2350 \mathrm{~km}$. 2005 FY9 and 2003 EL61 are among "scattered-near" objects (i.e. non-resonant, non-planet-crossing objects with Tisserand parameters less than 3, relative to Neptune) in the Deep Ecliptic Survey classification system (Elliot et al. 2005). 2005 FY9 has a perihelion distance, q, of 38.6 AU, an aphelion distance, Q, of $52 \mathrm{AU}$, a semi-major axis, a, of $45.3 \mathrm{AU}$, an inclination 
angle to the ecliptic, i, of $29^{\circ}$, and an eccentricity of 0.15. 2003 EL61 has $\mathrm{q}=35.1 \mathrm{AU}, \mathrm{Q}$ $=51 \mathrm{AU}, \mathrm{a}=43.1 \mathrm{AU}, \mathrm{i}=28^{\circ}$, and $\mathrm{e}=0.18$ For comparison, Pluto has $\mathrm{q}=29.7 \mathrm{AU}, \mathrm{Q}=$ $49 \mathrm{AU}, \mathrm{a}=39.5 \mathrm{AU}, \mathrm{i}=17^{\circ}$, and $\mathrm{e}=0.25$. At the time of observation, $2005 \mathrm{FY} 9$ and 2003 EL61 were both near aphelion.

\section{Observations}

We obtained spectra of 2005 FY9 and 2003 EL61 on 2006 March 5 UT with the 6.5meter MMT telescope on Mt. Hopkins, AZ, the Red Channel Spectrograph, and a $1200 \times$ 800 CCD. A $150 \mathrm{~g} \mathrm{~mm}^{-1}$ grating and a 1 arc sec slit width provided wavelength coverage of 5000 to $9500 \AA$ in first order, a dispersion of $6.38 \AA$ pixel $^{-1}$, and a fwhm resolution of 20.0 A. A LP495 blocking filter eliminated contamination from the second order.

There were high, thin cirrus clouds through most of the night and the seeing was $\sim 0.8$ arc sec. The KBOs were placed at the center of the slit and tracked at KBO rates.

We used the Image Reduction and Analysis Facility (IRAF) and standard procedures

(Massey et al. 1992) to calibrate and extract one-dimensional spectra from the two-dimensional spectral images. Specifically, the electronic bias of each image was removed by subtracting its overscan as well as a bias picture. Pixel to pixel sensitivity variations were removed from each image by dividing by a normalized twilight flatfield image. Extraction of onedimensional spectra from the images was done with the apall task in IRAF. HeNeAr spectra were used to correct for flexure and obtain an accurate wavelength calibration. Our wavelengths are accurate to $\sim$ one-tenth of a pixel or $\sim 0.7 \AA$. We removed telluric bands and Fraunhofer lines from the KBO spectra by observing the solar analog HD 112257 (Hardorp 1982) at airmasses very close to 2005 FY9 and 2003 EL61, and then then dividing the KBO spectra by the normalized solar analog spectra. Typically, the airmass difference between the KBOs and the solar analog was $\leq 0.05$. The 2005 FY9 and 2003 EL61 spectra in the analysis below are an average of four 10-minute and five 10-minute exposures, respectively.

\section{Results}

\subsection{FY9}

We plot our reflectance spectrum of 2005 FY9 in Figure 1 (bottom). The tick marks correspond to previously reported absorption maxima of $\mathrm{CH}_{4}$-ice (Table 1). The middle and top spectra in Figure 1 are of 2005 FY9 (Licandro et al. 2006) and Pluto (Grundy \& Fink 
1996). All spectra are normalized to 1 at $6500 \AA$. For the purpose of comparison, the Licandro et al. (2006) 2005 FY9 and Pluto spectra are offset by 0.4 and 0.8 , respectively. Clearly, 2005 FY9 exhibits much deeper $\mathrm{CH}_{4}$-ice bands than Pluto.

Our spectrum differs from the Licandro et al. (2006) spectrum in two ways. First, our spectrum exhibits additional subtle absorption bands at 5400, 5800, and $6000 \AA$. These bands have not been seen before in astronomical or laboratory spectra of $\mathrm{CH}_{4}$-ice. Because the bands are close to the wavelengths of gas phase $\mathrm{CH}_{4}$ bands and because there are strong $\mathrm{CH}_{4}$-ice bands at longer wavelengths in the spectrum of $2005 \mathrm{FY}$, we think the new bands are due to $\mathrm{CH}_{4}$-ice. In addition, it appears the band at $6200 \AA$ is $\mathrm{CH}_{4}$-ice as well. It is in the Licandro et al. (2006) spectrum, but they do not identify it as $\mathrm{CH}_{4}$-ice. Second, we find a continuum slope between 5500 and $6500 \AA$ of $10 \% / 1000 \AA$ while Licandro et al. (2006) find a slope of $13 \% / 1000 \AA$, with both spectra normalized to 1 at $6500 \AA$. We used HD 112257 , a G2V star, for our solar analog star whereas Licandro et al. (2006) used BS 4486, a G0V star, for their solar analog star. An examination of the $\mathrm{V}-\mathrm{R}$ colors for G0V and G2V stars suggests the slope difference is due to the use of different solar analog stars.

In order to place constraints on the $\mathrm{CH}_{4}$ grain size as well as the presence of any additional ice components on the surface of $2005 \mathrm{FY}$, we calculated model $\mathrm{CH}_{4}$-ice spectra. Our models use laboratory optical constants for pure $\mathrm{CH}_{4}$-ice at $30 \mathrm{~K}$ (Grundy et al. 2002) and arbitrary optical constants that absorb more at blue than red wavelengths, and thereby reproduce the observed reddish slope for the 2005 FY9 continuum. The ice and reddening agent were mixed in the model at the molecular level (i.e. by means of a weighted average of the ice and reddening agent optical constants). We used Hapke theory (Hapke 1981, 1993) to transform the optical constants into reflectance spectra. We used Hapke parameters $h$ $=0.1, \mathrm{~B}_{o}=0.8, \bar{\theta}=30^{\circ}$, and $\mathrm{P}(\mathrm{g})=$ a two component Henyey-Greenstein function with $80 \%$ in the forward scattering lobe and $20 \%$ in the back scattering lobe and both lobes having asymmetry parameter 0.63 . These values are comparable to numbers used previously to model the surface of Pluto (Grundy \& Buie 2001).

Figure 2 compares the spectrum of 2005 FY9 (black line) and a Hapke model with a grain size of $0.55 \mathrm{~cm}$ (red line). Although the model fits the core of the $8897 \AA$ band, it has too little absorption at all the other bands. Next, we fit the core of the weaker $7296 \AA$ band with a $1.8 \mathrm{~cm}$ grain size. Then, we fit the core of the even weaker $8000 \AA$ complex with a $2.5 \mathrm{~cm}$ grain size. These models illustrate a trend, weaker bands (which see deeper into the surface) aways seem to need bigger grain sizes, or equivalently, greater optical path lengths in $\mathrm{CH}_{4}$-ice.

From the above discussion, it is clear a single grain size will not fit the observations. In Figure 3, we present a Hapke model that uses two grain sizes, $6 \mathrm{~cm}$ (97\% by volume) and 
$0.1 \mathrm{~cm}(0.03 \%$ by volume), in an intimate mixture. The inability of the two-grain size model to fit the $\mathrm{CH}_{4}$-ice band at wavelengths less than $7000 \AA$ in Figure 3 is due to the lack of laboratory optical constants. We note that our two-grain size model is not a unique solution. Although this model fits the data, other models (such as courser grains underneath a coating of finer grains) would probably fit the data just as well. In addition, it is important to realize that grain sizes larger than $1 \mathrm{~cm}$ are probably a measure of the spacing of fractures or voids in the solid surface rather than indicating a surface covered by golf-ball sized particles.

Although we can fit the shape and depth of the absorptions, there is a small but significant difference between our models and the telescope data. Specifically, the maxima of the $\mathrm{CH}_{4}$ absorption bands in the spectrum of $2005 \mathrm{FY} 9$ reside blueward of the maxima in the pure $\mathrm{CH}_{4}$ Hapke models. In Figure 4, we present a small portion of Figure 3 centered on the $7296 \AA$ band to illustrate the shift. Such a shift is important because laboratory experiments show that the 8897,8968 , and $9019 \AA$ bands in spectra of pure $\mathrm{CH}_{4}$-ice are shifted blueward by $27 \AA$ in a $\mathrm{CH}_{4} / \mathrm{N}_{2}=0.8 \%$ mixture (Quirico \& Schmitt 1997). Another experiment finds the $8897 \AA$ band shifts $\sim 17 \AA$ in a $\mathrm{CH}_{4} / \mathrm{N}_{2}=20 \%$ ice mixture (Grundy \& Fink 1993).

A cross correlation experiment provides a way to quantify the apparent shift in Figure 3. Specifically, we wrote a Fortran program to shift the model spectrum from -25 to +25 $\AA$ in $1 \AA$ steps. For each shift, the program finds the difference between the data and model, i.e. it calculates $\left(y_{d, i}-y_{m, i}\right)$ where $y_{d, i}$ and $y_{m, i}$ represent the ordinate values of the data and the model spectra at wavelength $i$. Then, the program sums the squares of the difference over all N wavelength points between 7000 and $9300 \AA$. In other words, we calculate

$$
\Delta_{\text {shift }}=\sum_{i}^{N}\left(y_{d, i}-y_{m, i}\right)^{2} .
$$

In Figure 5, we present a plot of the sums of the squares of the differences, i.e. $\Delta_{\text {shift }}$, as function of shift. We find a well defined minimum at $3.25 \AA$.

What is the uncertainty in the $3.25 \AA$ measurement? We note that HeNeAr spectra enable us to calibrate the wavelengths in the 2005 FY9 spectrum to an uncertainty of $\sim$ $1 / 10$ of a pixel or $\sim 0.7 \AA$. Specifically, we find the average difference between the centroids of airglow lines in our data and the corresponding laboratory values is $0.7 \AA$. A larger source of uncertainty comes from the noise in the spectra and the broadness of the cross correlation minimum in Figure 5. Propagation of the noise in the data and model spectra for the $3 \AA$ shift through equation (1) gives an ordinate error bar for the minimum point $(3.25,2.218)$ in Figure 5 of 0.042 . As a result, the uncertainty in our shift measurement is defined by $\Delta_{\text {shift }}<2.218+0.042=2.260$ (i.e. the section of the curve below the dashed line in Figure 
5), corresponding to shifts between 1 and $5.5 \AA$. In short, we find the $\mathrm{CH}_{4}$-ice bands in the spectrum of 2005 FY9 are blueshifted relative to the model Hapke spectrum by $3.25 \pm 2.25$ $\AA$.

Licandro et al. (2006) gave blueshifts of 2 - $6 \AA$ for $\mathrm{CH}_{4}$ bands in their Table 1, but they questioned the reality of the shifts because they are so small. Our independent measurement of shifts with similar magnitude bolsters the case for the reality of the small shifts. In fact, when we take the Licandro et al. (2006) data and do a cross correlation experiment between our spectrum and their spectrum, we find no measurable shift between the spectra; but when we perform a cross correlation experiment between their spectrum and the Hapke model, we find their $\mathrm{CH}_{4}$-ice bands are blue shifted $2 \AA$ relative to the model. Our spectrum and the Licandro et al. (2006) spectrum are consistent.

\subsection{EL61}

We plot our reflectance spectrum of 2003 EL61 in Figure 6. The continuum has a slope of essentially zero, consistent with $\mathrm{B}-\mathrm{V}=0.63 \pm 0.03$ and $\mathrm{V}-\mathrm{R}=0.34 \pm 0.02$ (Rabinowitz et al. 2006). Evidently, tholins are not present on the surface of 2003 EL61 at an abundance seen on 2005 FY9. (Recall that both objects were observed on the same night and compared against the same solar analog spectrum.) We find no evidence of $\mathrm{CH}_{4^{-}}$ ice bands, but we can set an upper limit on the thickness of a global glaze of $\mathrm{CH}_{4}$-ice by applying Beer's Law,

$$
\left(\frac{I}{I_{o}}\right)_{\lambda}=e^{-2 a_{\lambda} t}
$$

to the continuum near the $8897 \AA$ band. In the above equation, $\mathrm{t}$ is the thickness of the glaze, $\mathrm{a}_{\lambda}$ are the Lambert absorption coefficients for $\mathrm{CH}_{4}$-ice (Grundy et al. 2002), the factor of two comes about because light passes through the glaze once on the way in and once on the way back out, $\left(\mathrm{I} / \mathrm{I}_{o}\right)_{\lambda}$ is the ratio of light leaving the glaze to the light incident on the glaze. For $\left(\mathrm{I} / \mathrm{I}_{o}\right)_{\lambda=8897}=0.97$, we find $\mathrm{t}<0.3 \mathrm{~mm}$.

$\mathrm{H}_{2} \mathrm{O}$-ice bands are seen in near-infrared spectra of 2003 EL61 (Barkume et al. 2006). Therefore, it is reasonable to look for evidence of solid-state photolytic or radiolytic chemistry (Johnson 1990; Johnson \& Quickenden 1997; Delitsky \& Lane 1997) by seeing if $\mathrm{O}_{2}$-ice is present on the surface of 2003 EL61. In Figure 6, we superimpose the 5773 and $6275 \AA$ $\mathrm{O}_{2}$-ice bands in spectra of Ganymede (Calvin \& Spencer 1997) on top of our 2003 EL61 spectrum. There is a tantalizing dip at $5773 \AA$ in the spectrum of 2003 EL61, but no dip at 
the weaker $6275 \AA$ band. The continuum signal precision at the position of these bands is about 150 and so if the $5773 \AA$ band is real, it would take a continuum signal precision of about 300 to detect the $6275 \AA$ band.

We estimate an upper limit to the thickness of a global glaze of $\mathrm{O}_{2}$-ice by assuming the possible feature at $5773 \AA$ is real and then applying Beer's Law as we did for $\mathrm{CH}_{4}$-ice. For $\left(\mathrm{I} / \mathrm{I}_{o}\right)_{\lambda=5573}=0.99$ and $\mathrm{a}_{\lambda=5773}=5.5 \mathrm{~cm}^{-1}$, we find $\mathrm{t}<0.01 \mathrm{~mm}$. Our value for $\mathrm{a}_{\lambda=5773}$ comes from an integrated absorption coefficient of $1.1 \times 10^{3} \mathrm{~cm}^{-2}$ and a band fwhm of $\sim$ $200 \mathrm{~cm}^{-1}$ (Landau et al. 1962).

\section{Conclusions}

Our spectrum of $2005 \mathrm{FY} 9$ exhibits strong $\mathrm{CH}_{4}$-ice bands in agreement with the spectrum of Licandro et al. (2006). From a comparison of our spectrum and a Hapke model, we find the $2005 \mathrm{FY} \mathrm{CH}_{4}$-ice bands are shifted $3.25 \pm 2.25 \AA$ blueward relative to the positions of pure $\mathrm{CH}_{4}$-ice bands. The shift could be due to the presence of another ice-component, possibly $\mathrm{N}_{2}$-ice, $\mathrm{CO}$-ice, or Ar. Future higher resolution spectra of the individual $\mathrm{CH}_{4}$ bands should determine if the weaker bands which penetrate deeper into the surface exhibit different shifts than the stronger bands which do not penetrate as deep into the surface. Such observations could provide a technique to measure the $\mathrm{CH}_{4}$ concentration relative to the other ice component as a function of depth below the surface of 2005 FY9. In addition, it is highly desirable to obtain a measure of the shift as a function of rotational longitude on $2005 \mathrm{FY} 9$ for a particular $\mathrm{CH}_{4}$-ice band. Such a technique could provide a measure of inhomogeneity on the surface of 2005 FY9. Grundy \& Fink (1996) made such measurements for the $8897 \AA$ band on Pluto and found that the blueshift varied from 0 to $10 \AA$ over the surface.

On Pluto, it appears $\mathrm{N}_{2}$ is sufficiently mobile to form textures that allow incoming photons to travel several centimeters through the very transparent, polycrystalline $\mathrm{N}_{2}$-ice before the photons are scattered off inclusions or grain boundaries. The long pathlengths make it possible for a small amount of $\mathrm{CH}_{4}$ relative to $\mathrm{N}_{2}$ to give deep $\mathrm{CH}_{4}$ absorption bands. The mobility of $\mathrm{CO}$ is only a little less than $\mathrm{N}_{2}$, and the mobility of Ar is only a little less than CO. Therefore, it is possible that CO-ice or Ar-ice could provide the host matrix for long optical pathlengths. It is also possible the host matrix is a combination of $\mathrm{N}_{2}, \mathrm{CO}$, and Ar. A suite of laboratory experiments that measure the shifts of $\mathrm{CH}_{4}$ absorption maxima for different concentrations of $\mathrm{CH}_{4}$ relative to $\mathrm{N}_{2}, \mathrm{CO}$, and Ar would be highly valuable for the future interpretation of observational data. 
Our spectrum of 2003 EL61 is remarkably featureless. There is a hint of a band at $5773 \AA$ possibly due to $\mathrm{O}_{2}$-ice. Future work will attempt to double the signal precision of the continuum to at least 300, which is the level that appears to be required for any possible detection of the weaker $\mathrm{O}_{2}$-ice band at $6275 \AA$. The detection of $\mathrm{O}_{2}$-ice in significant quantities on KBOs could be of considerable interest in the distant future as a source of spacecraft fuel.

\section{Acknowledgments}

SCT, WR, and KM gratefully acknowledge support from NASA Planetary Astronomy grant NNG06G138G to Northern Arizona University and the University of Oklahoma. WMG gratefully acknowledges support from NASA Planetary Geology and Geophysics grant NNG04 G172G to Lowell Observatory. GJC gratefully acknowledges support from Fordham University. We thank Steward Observatory for the allocation of telescope time on the MMT. We thank Dr. J. Licandro for providing us with his spectrum of 2005 FY9. We thank an anonymous referee for a careful review of our manuscript. 


\section{REFERENCES}

Barkume, K. M., Brown, M. E., and Schaller, E. L. 2006, ApJ, 640, L87.

Brown, M. E., Barkume, K. M., Blake, G. A., Schaller, E. L., Rabinowitz, D. L., Roe, H. G., \& Trujillo, C. A. 2006, AJ, submitted.

Brown, M. E., Trujillo, C. A., \& Rabinowitz, D. L. 2005, ApJ, 635, L97

Brown, R. H., Cruikshank, D. P., \& Pendleton, Y. 1999, ApJ, 519, L101

Calvin, W. M., \& Spencer, J. R. 1997, Icarus, 130, 505.

Cruikshank, D. P., Pilcher, C. B., \& Morrison, D. 1976, Science, 194, 835

Cruikshank, D. P., Roush, T. L., Owen, T. C., Geballe, T. R., de Bergh, C., Schmitt, B., Brown, R. H. and Bartholomew, M. J. 1993, Science, 261, 742

Delitsky, M. L., \& Lane, A. L. 1997, J. Geophys. Res., 102, 16385

Doressoundiram, A., Tozzi, G. P., Barucci, M. A., Boehnhardt, H., Fornasier, S. \& Romon J. 2003, AJ, 125, 2721

Elliot, J. L., Kern, S. D., Clancy, K. B., Gulbis, A. A. S., Millis, R. L., Buie, M. W., Wasserman, L. H., Chiang, E. I., Jordan, A. B., Trilling, D. E., and Meech, K. J. 2005, AJ, 129, 1117

Fink, U., Smith, B. A., Johnson, J. R., Reitsema, H. J., Benner, D. C., \& Westphal, J. A. 1980, Icarus, 44, 62

Fornasier, S., Dotto, E., Barucci, M. A., \& Barbieri, C. 2004a, A\&A, 422, 43

Fornasier, S., Doressoundiram, A., Tozzi, G. P., Barucci, M. A., Boehnhardt, H., de Bergh, C., Delsanti, A., Davies, J., \& Dotto, E. 2004b, A\&A, 421, 353

Grundy, W. M., \& Buie, M. W. 2001, Icarus, 153, 248

Grundy, W. M., \& Fink, U. 1993, Pluto \& Charon Conference, Flagstaff, AZ, July 6-9, 1993

Grundy, W. M. \& Fink, U. 1996, Icarus, 124, 329.

Grundy, W. M., Schmitt, B., and Quirico, E. 2002, Icarus, 155, 486.

Hapke, B. 1981, J. Geophys. Res., 86, 4571. 
Hapke, B. 1993, Combined Theory of Reflectance and Emittance Spectroscopy (New York: Cambridge Univ. Press)

Hardorp, J. 1982, A\&A, 105, 120

Jewitt, D. C., \& Luu, J. 2004, Nature, 432, 731

Johnson, R. E. 1990, Energetic Charged-Particle Interactions With Atmospheres and Surfaces. (New York: Springer-Verlag)

Johnson, R. E., \& Quickenden, T. I. 1997, J. Geophys. Res., 102, 10985

Landau, A., Allin, E. J., \& Welsh, H. L. 1962, Spectrochimica Acta, 18, 1.

Licandro, J., Oliva, E., \& Di Martino, M. 2001, A\&A, 373, L29

Licandro, J., Pinilla-Alonso, N., Pedani, M., Oliva, E., Tozzi, G. P., and Grundy, W. M. 2006, A\&A, 445, L35.

Luu, J. \& Jewitt, D. 1996, AJ, 112, 2310

Massey, P., Valdes, F., \& Barnes, J. 1992, in A User's Guide to Reducing Slit Spectra With IRAF

Quirico, E., \& Schmitt, B. 1997, Icarus, 127, 354

Rabinowitz, D. L., Barkume, K., Brown, M. E., Roe, H., Schwartz, M., Tourtellotte, S., and Trujillo, C. 2006, ApJ, 639, 1238. 


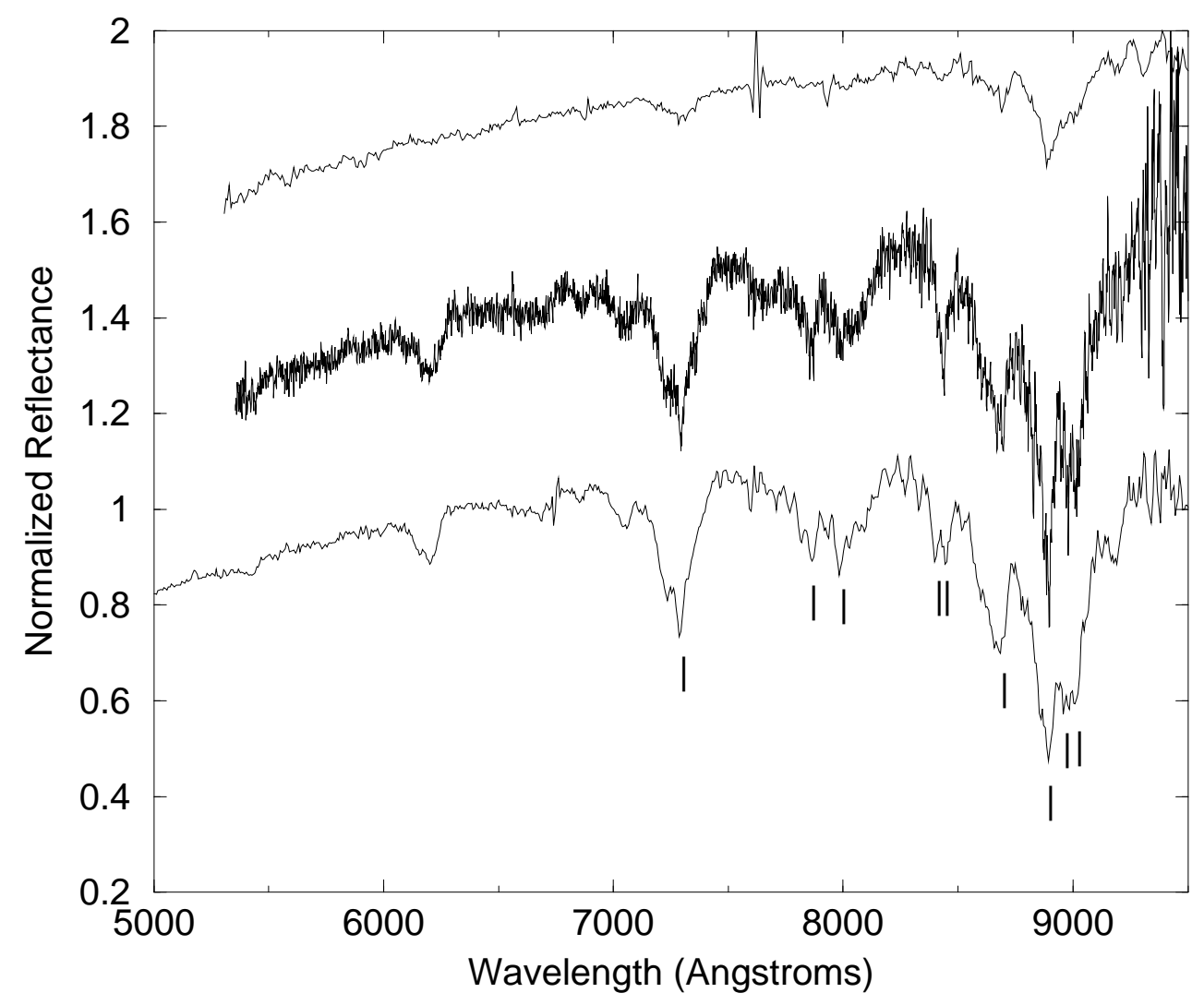

Fig. 1.- Our normalized reflectance spectrum of 2005 FY9 (bottom). Tick marks indicate the position of previously reported $\mathrm{CH}_{4}$-ice bands, see Table 1 . The middle spectrum is also of 2005 FY9 (Licandro et al. 2006). The top spectrum is of Pluto (Grundy \& Fink 1996). All spectra are normalized to 1 at $6500 \AA$. The Licandro et al. (2006) and Pluto spectra are offset by 0.4 and 0.8, respectively. Clearly, 2005 FY9 has deeper $\mathrm{CH}_{4}$-ice bands than Pluto. Previously unreported $\mathrm{CH}_{4}$-ice bands appear near 5400, 5800, and $6200 \AA$ in our spectrum of 2005 FY9. 


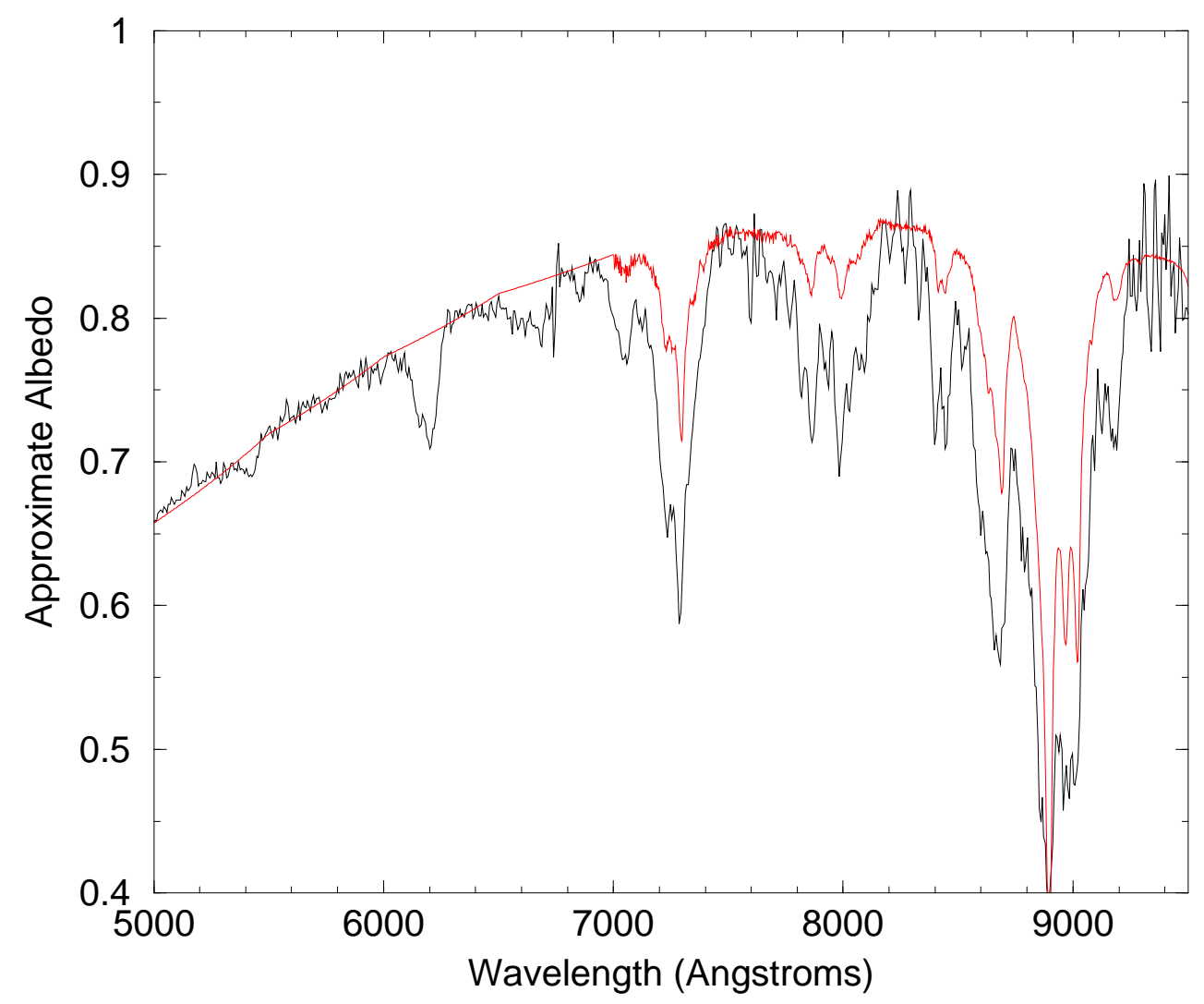

Fig. 2.- Approximate albedo spectrum of 2005 FY9 (black line) and a Hapke model with a reddening agent and a $\mathrm{CH}_{4}$-ice grain size of $0.55 \mathrm{~cm}$ (red line). We assume an albedo at $6500 \AA$ of 0.8 . The model fits the core of the $8897 \AA$ band, but has too little absorption at all other bands. There are no laboratory absorption coefficients to fit $\mathrm{CH}_{4}$ bands with $\lambda<$ $7000 \AA$. 


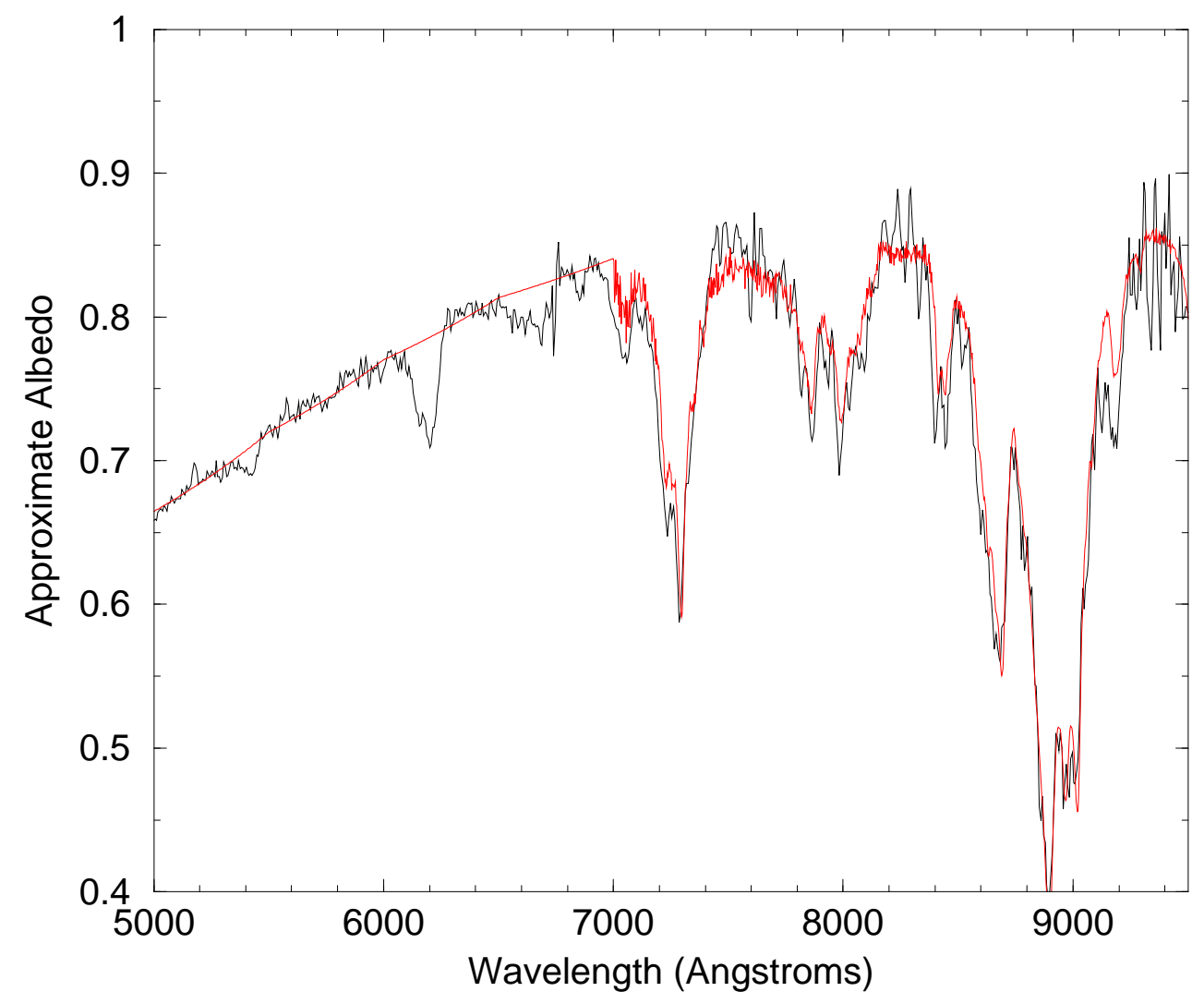

Fig. 3.- Approximate albedo spectrum of 2005 FY9 (black line) and a Hapke model with a reddening agent and two $\mathrm{CH}_{4}$-ice grain sizes, $6 \mathrm{~cm}$ and $0.1 \mathrm{~cm}$ (red line). We assume an albedo at $6500 \AA$ of 0.8 . Grains sizes larger than a cm probably provide a measure of the spacing between fractures or voids in the surface rather than indicating a surface covered with golf-ball sized particles. There are no laboratory absorption coefficients to fit $\mathrm{CH}_{4}$ bands with $\lambda<7000 \AA$ 


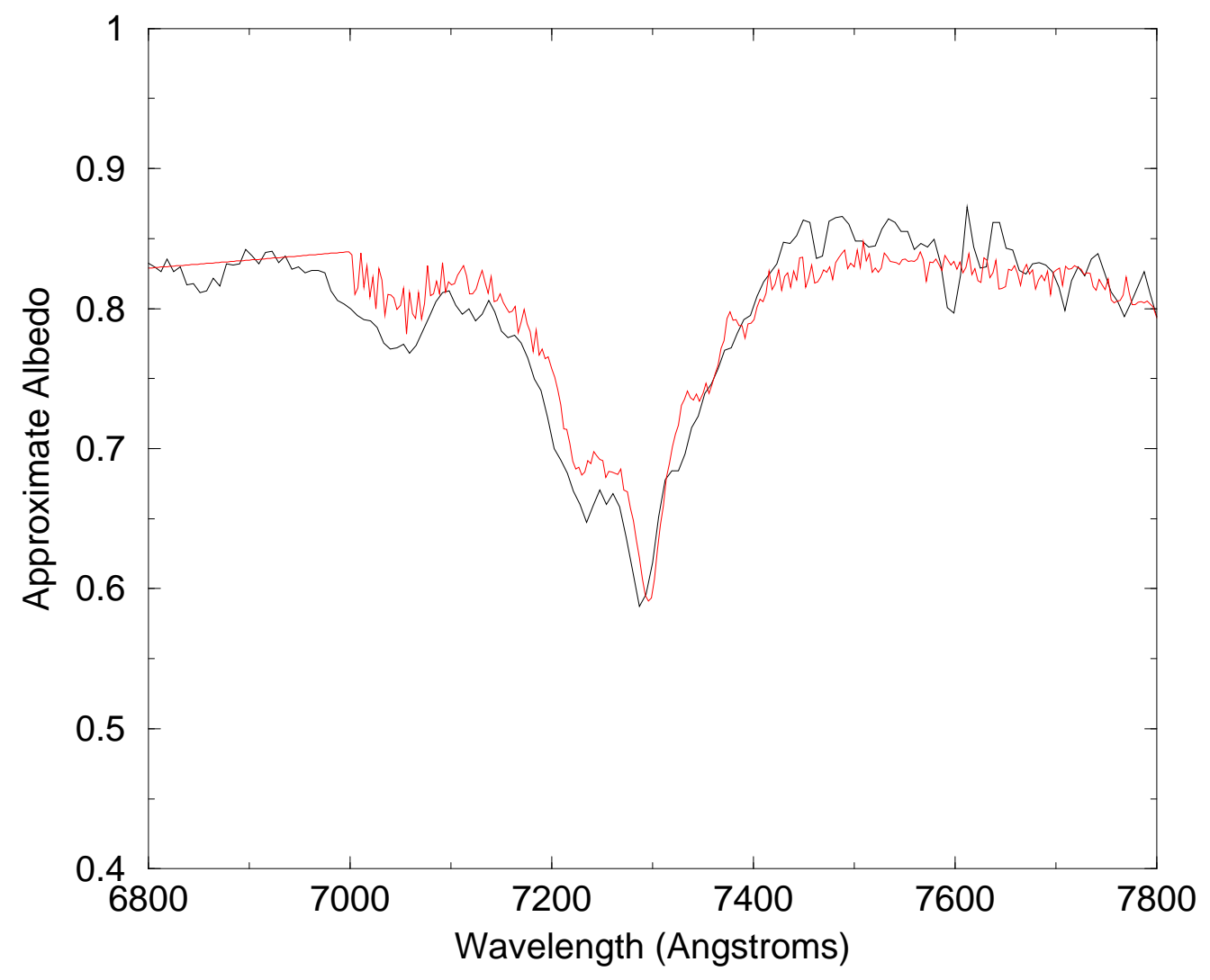

Fig. 4. - The minimum of the $7296 \AA \mathrm{CH}_{4}$-ice band in $2005 \mathrm{FY} 9$ (black line) is blueshifted relative to the minimum of the pure $\mathrm{CH}_{4}$-ice Hapke model (red line). The other bands in 2005 FY9 show a similar shift. The shift may be due to the presence of another ice component on the surface of 2005 FY9. 


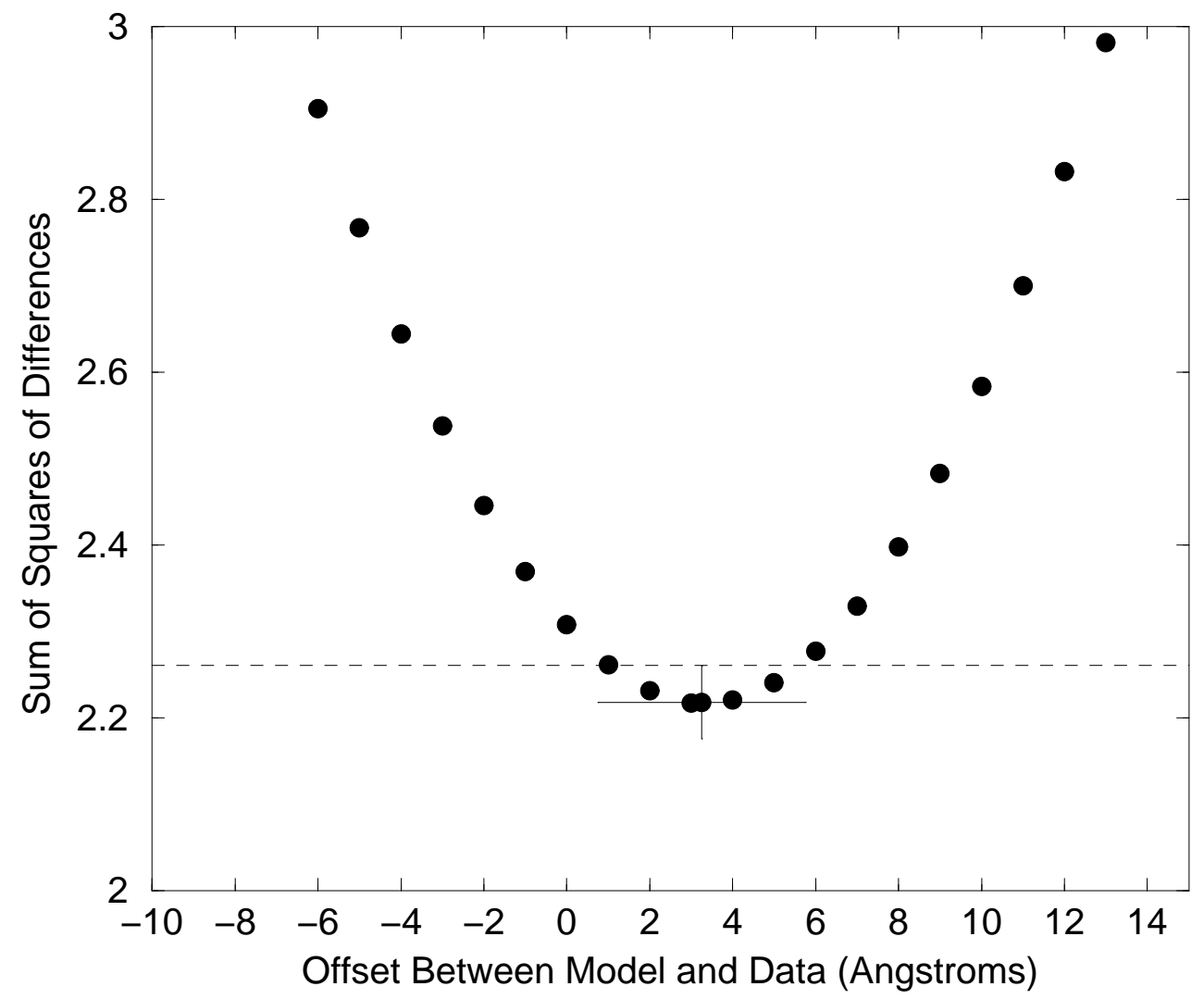

Fig. 5.- Result of cross-correlation experiment between the spectra of 2005 FY9 and the pure $\mathrm{CH}_{4}$-ice Hapke model in Figure 3. The difference between the two spectra reach a minimum at a $3.25 \pm 2.25 \AA$ blueshift of the data relative to the model. 


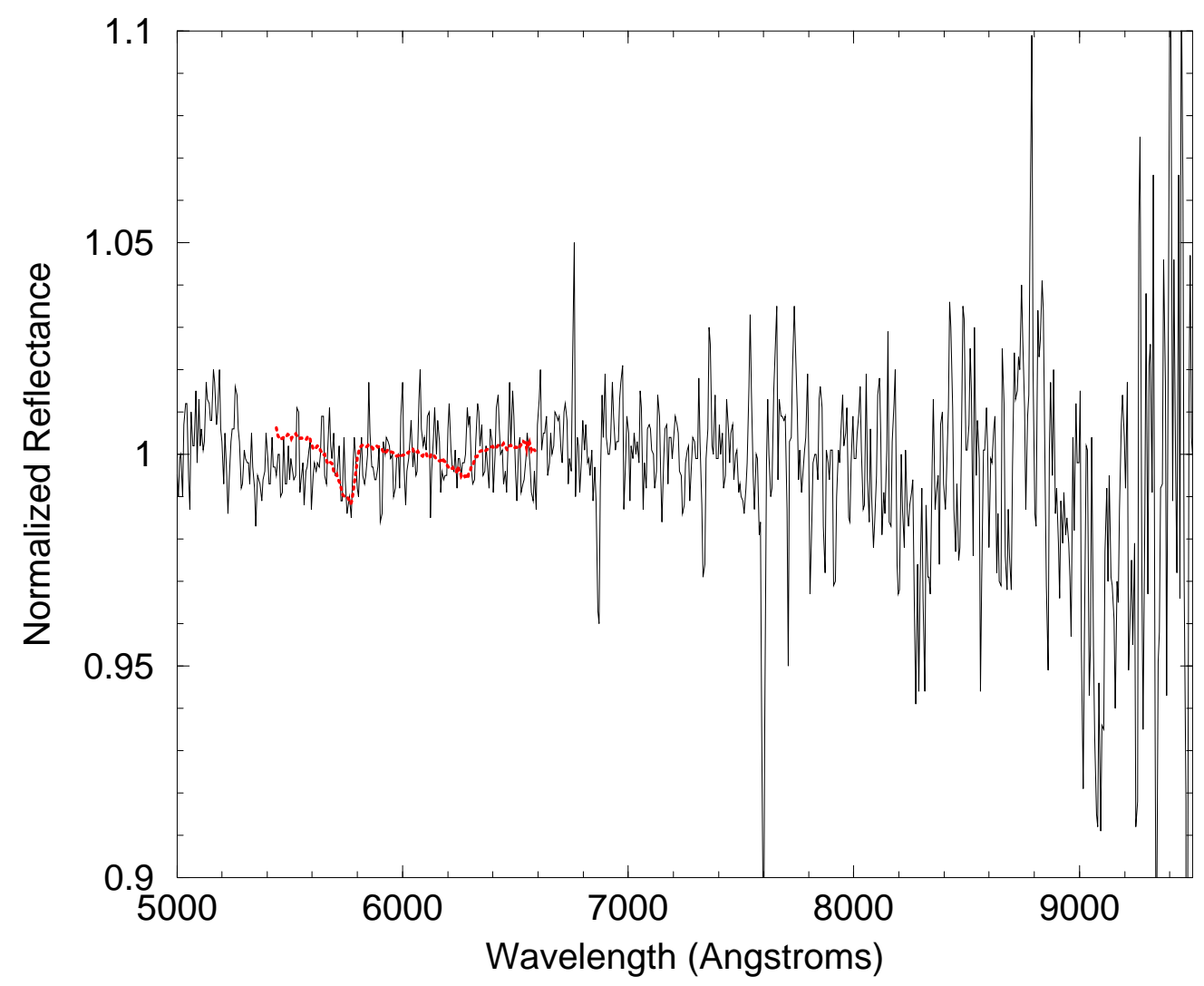

Fig. 6.- Normalized reflectance spectrum of 2003 EL61 (black line) and the spectral ratio of Ganymede to Callisto (Calvin \& Spencer 1997) showing $\mathrm{O}_{2}$-ice bands at 5773 and 6275 $\AA$ (red line). There is a tantalizing dip in the spectrum of 2003 EL61 at the position of the $5773 \AA$ band; however, a much higher continuum signal precision is necessary to test for the presence of the weaker $6275 \AA$ band. 
Table 1. Laboratory Wavelengths and Frequencies of $\mathrm{CH}_{4}$-Ice Absorption Maxima

\begin{tabular}{lcc}
\hline \hline \multicolumn{1}{c}{ Transition } & Wavelength $(\AA)$ & Wavenumber $\left(\mathrm{cm}^{-1}\right)$ \\
\hline $3 \nu_{1}+4 \nu_{4}$ & 7296 & 13706 \\
$3 \nu_{3}+3 \nu_{4}$ & 7862 & 12719 \\
$3 \nu_{1}+3 \nu 4$ & 7993 & 12511 \\
$4 \nu_{3}$ & 8415 & 11884 \\
$\nu_{1}+3 \nu_{3}$ & 8442 & 11846 \\
$3 \nu_{3}+2 \nu_{4}$ & 8691 & 11506 \\
$2 \nu_{1}+\nu_{3}+2 \nu_{4}$ & 8897 & 11240 \\
$3 \nu_{1}+2 \nu_{4}$ & 8968 & 11151 \\
$2 \nu_{3}+4 \nu_{4}$ & 9019 & 11088 \\
\hline
\end{tabular}

${ }^{\text {a Pure }} \mathrm{CH}_{4}$ Ice I at $30 \mathrm{~K}$ (Grundy et al. 2002) 